This item was submitted to Loughborough's Research Repository by the author.

Items in Figshare are protected by copyright, with all rights reserved, unless otherwise indicated.

\title{
Measuring student motivation for physical education: examining the psychometric properties of the Perceived Locus of Causality Questionnaire and the Situational Motivation Scale
}

\section{PLEASE CITE THE PUBLISHED VERSION}

http://dx.doi.org/10.1016/j.psychsport.2010.11.003

\section{PUBLISHER}

(C) Elsevier Ltd

\section{VERSION}

AM (Accepted Manuscript)

\section{LICENCE}

CC BY-NC-ND 4.0

\section{REPOSITORY RECORD}

Lonsdale, Chris C., Catherine M. Sabiston, lan M. Taylor, and Nikos Ntoumanis. 2019. "Measuring Student Motivation for Physical Education: Examining the Psychometric Properties of the Perceived Locus of Causality Questionnaire and the Situational Motivation Scale". figshare. https://hdl.handle.net/2134/15374. 
This item was submitted to Loughborough's Institutional Repository (https://dspace.lboro.ac.uk/) by the author and is made available under the following Creative Commons Licence conditions.

\section{creative
commons}

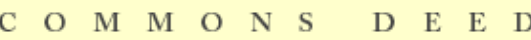

Attribution-NonCommercial-NoDerivs 2.5

You are free:

- to copy, distribute, display, and perform the work

Under the following conditions:

Attribution. You must attribute the work in the manner specified b the author or licensor.

Noncommercial. You may not use this work for commercial purposes.

No Derivative Works. You may not alter, transform, or build upon this work.

- For any reuse or distribution, you must make clear to others the license terms of this work.

- Any of these conditions can be waived if you get permission from the copyright holder.

Your fair use and other rights are in no way affected by the above.

This is a human-readable summary of the Leqal Code (the full license).

\section{Disclaimer 만}

For the full text of this licence, please go to: http://creativecommons.org/licenses/by-nc-nd/2.5/ 
Measuring Motivation for Physical Education... 1

Measuring Student Motivation for Physical Education: Examining the Psychometric Properties of the Perceived Locus of Causality Questionnaire and the Situational Motivation Scale

DATE OF SUBMISSION: August 18, 2010 


\begin{abstract}
Objectives: The purpose of this study was to evaluate, within a cross-cultural context, the psychometric properties of scores derived from the Perceived Locus of Causality Questionnaire (PLOCQ) and the Situational Motivation Scale (SIMS). Both questionnaires are grounded in self-determination theory and are commonly employed in physical education research.
\end{abstract}

Method: Secondary school students from the United Kingdom (UK; $n=300$, mean age = 13.71) and Hong Kong (HK; $n=342$, mean age $=15.34$ years) completed both questionnaires prior to a physical education lesson.

Results: Internal consistency analyses, as well as single and multi-group confirmatory factor analyses produced evidence that largely supported the reliability and validity of PLOCQ and SIMS scores in the UK sample. However, the analyses indicated some areas of concern regarding the internal consistency of the external and introjected regulation PLOCQ items in the HK sample. Also, identified regulation and intrinsic motivation constructs were not distinguishable by youth in either culture in either questionnaire. Finally, compared with the UK, students in HK interpreted the SIMS external regulation items to be more selfdetermined.

Conclusions: Researchers interested in studying contextual and situational motivation in UK physical education classes should, in general, feel confident in using the PLOCQ and the SIMS, respectively. However, our results highlight some important difficulties in the measurement of contextual and situational motivation in HK Chinese students. Further research is needed to better understand how students from different cultures respond to items intended to tap controlling forms of motivation.

Key Words: self-determined motivation, intrinsic motivation, extrinsic motivation, amotivation, external regulation, introjected regulation, identified regulation 


\section{Measuring Student Motivation for Physical Education: Examining the Psychometric} Properties of the Perceived Locus of Causality Questionnaire and the Situational

\section{Motivation Scale}

Motivation is an important variable to consider in the physical education (PE) context, as adaptive types of motivation have been associated with intentions to exercise (Standage, Duda, \& Ntoumanis, 2003), step counts during PE classes (Lonsdale, Sabiston, Raedeke, Ha, \& Sum, 2009) and physical activity during leisure-time (Gordon-Larsen, McMurray, \& Popkin, 2000; Hagger, Chatzisarantis, Culverhouse, \& Biddle, 2003). Self-determination theory (SDT; Ryan \& Deci, 2002) has been widely used to study motivation within the PE context (see review by Ntoumanis \& Standage, 2009). Nonetheless, there has been limited attention paid to the validity of scores derived from popular SDT-based measures of students' motivation for PE.

Filling this research gap is important if SDT is to continue as a significant framework to study motivation in PE. The primary purpose of the current study was to evaluate the psychometric properties of scores derived from two instruments, the Perceived Locus of Causality questionnaire (PLOCQ; Goudas, Biddle, \& Fox, 1994) and the Situational Motivation Scale (SIMS; Guay, Vallerand, \& Blanchard, 2000), both of which are grounded in SDT and are commonly employed in research in the PE context (Lonsdale, et al., 2009; Ntoumanis, 2001, 2005; Prusak, Treasure, Darst, \& Pangrazi, 2004; Taylor \& Ntoumanis, 2007). A second aim was to investigate the cross-cultural equivalence of scores derived from these measures in the United Kingdom (UK) and the Chinese 'special administrative region' of Hong Kong (HK). Examining the cross-cultural validity of scores derived from popular motivation questionnaires is an important research objective, given the proposed applicability of SDT tenets across cultures (Deci, et al., 2001). 


\section{Self-Determination Theory}

Deci and Ryan (1985) proposed that different types of motivation lie on a continuum according to their level of self-determination. The most self-determined motivation is intrinsic motivation, which refers to partaking in an activity because of interest and/or enjoyment. Next, extrinsic motivation is generally defined as the participation in an activity because of a goal distinct from the activity itself and is conceptualized according to four behavioral regulations. Integrated regulation, the most self-determined form of extrinsic motivation, refers to the pursuit of an activity because it is consistent with one's values and sense of self. Identified regulation refers to participating in an activity because one values its outcomes, whereas introjected regulation refers to doing an activity because of internal pressures such as guilt, shame, or ego protection/enhancement. The least self-determined form of extrinsic motivation is external regulation, which refers to the pursuit of an activity because of external coercive pressures or rewards. Finally, amotivation is defined as the absence of intrinsic or extrinsic motivation and, thus, the absence of self-determination (Ryan and Deci, 2002). An amotivated individual perceives no worthwhile reasons for participation.

The self-determination continuum is proposed to have a simplex-like structure, whereby adjacent regulations (e.g., intrinsic motivation and identified regulation) should be more strongly and positively related with each other, whereas more distal regulations (e.g., intrinsic motivation and amotivation) are expected to be unrelated or negatively correlated with each other (Ryan \& Connell, 1989). Chatzisarantis, Hagger, Biddle, Smith, and Wang (2003) found some support, via meta analysis, for the simplex-like structure in sport, leisure, and PE contexts. Nevertheless, there is a need to examine the simplex-like structure of scales purported to measure PE-specific motives (as opposed to motives across diverse PA-related contexts) within distinct cultural contexts. 
From an SDT perspective, motivation is also conceptualised to operate at three different levels of generality (Vallerand, 2001) - situational, contextual, and global. The situational level refers to motivation towards a specific activity at a particular point in time; for example, one's motivation to play basketball during a given PE class. Contextual motivation refers to one's reasons for participating in a more diverse set of related behaviors across a period of time; for example, one's motivation to take part in PE lessons more generally. Global motivation refers to an individual's tendency to be motivated in an intrinsic, extrinsic, or amotivated way across different life contexts. Contextual and situational motives are most often measured in PE research to assess stable and more transient motivation in PE, respectively.. Two instruments often employed to measure motivation at these two levels are the PLOCQ (Goudas, et al., 1994) and the SIMS (Guay, et al., 2000), which have not yet been explored within a cross-cultural context.

Contextual motivation towards PE is often measured using the PLOCQ. Goudas et al. (1994) developed this scale by adapting items from the Self-Regulation Questionnaire (Ryan \& Connell, 1989) to reflect intrinsic motivation, identified regulation, introjected regulation, and external regulation, as well as items from the amotivation subscale of the Academic Motivation Scale (Vallerand, et al., 1992). The PLOCQ does not include a measure of integrated regulation. Previous research has generally supported the reliability and validity of PLOCQ subscale scores (Ntoumanis, 2001, 2005; Standage, Duda, et al., 2003; Taylor \& Ntoumanis, 2007). Nonetheless, there has been some concern regarding the internal consistency of the introjected regulation scores (e.g., $\alpha=.64$, Ntoumanis, 2005) as well as the discriminant validity of the identified regulation and intrinsic motivation scores (e.g., $\Phi=$ .99, Standage, Duda, \& Ntoumanis, et al., 2005).

Situational motivation towards PE is typically measured using the SIMS (Guay et al., 2000). The SIMS contains items intended to measure intrinsic motivation, identified 
regulation, external regulation and amotivation. The SIMS was designed to be a brief measure and does not include an introjected or integrated regulation subscale. This instrument was not developed specifically for PE, but initial evidence from this context has supported the internal consistency and factorial validity of scores derived from a PE-modified version of the SIMS (Standage et al., 2003).

Careful instrument development and ongoing tests of validity and reliability are critical if researchers are to be confident that they have measured what they had intended to measure (Messick, 1995). With this in mind, Marsh (1998) has advocated a two step approach in which 'within-network' aspects of validity (e.g., factorial validity) are examined prior to 'between-network’ aspects of validity (e.g., convergent validity). To date, evidence regarding the validity of PLOCQ and SIMS scores has been limited to within-network investigations (Ntoumanis, 2001; Standage, Treasure, Duda, \& Prusak, 2003). In this study we sought to not only replicate previous investigations of within-network validity, but to also extend previous findings by examining between-network validity. Based on the proposed relationships outlined in Vallerand's (2001) hierarchical motivational model, we investigated convergent validity by examining correlations between factors from the PLOCQ and factors from the SIMS. Convergent validity would be supported if latent factors representing identical constructs at different levels of generality, or constructs similar in their level of selfdetermination, were more strongly correlated than factors from measures intended to represent dissimilar constructs (i.e., constructs that are more distal on the self-determination continuum). For example, we hypothesized that the amotivation factor from the PLOCQ would be more strongly, and positively, correlated with the SIMS amotivation factor than with factors from any other SIMS subscale. Furthermore, we expected that the PLOCQ amotivation factor would be more strongly, and positively, related to the SIMS external regulation factor than the SIMS identified regulation factor. 
Our second purpose was to investigate the cross-cultural equivalence of scores derived from both the PLOCQ and the SIMS. These measures have been used extensively to study PE motivation in Western countries, especially in the United Kingdom (UK) (e.g., Ntoumanis, 2001; 2005; Standage et al., 2003; Taylor \& Ntoumanis, 2007). However, there is only limited evidence of research employing these scales in non-Western nations (Hagger, Chatzisarantis, Barkoukis, Wang, \& Baranowski, 2005; Wang \& Liu, 2007). This dearth of research is unfortunate given the controversy surrounding the importance of selfdetermination across cultures (Chirkov, Ryan, Kim, \& Kaplan, 2003; Iyengar \& Lepper, 1999). Specifically, it has been suggested that self-determination is a socially constructed value embedded in Western societies, such as UK, that is synonymous with individualism and independence. Thus, self-determination may not be important for individuals' well-being in collectivist cultures, such as Hong Kong (HK), where interdependence and group conformity may be more central (Markus \& Kitayama, 1991). These proposals are contrary to SDT tenets. Specifically, advocates of SDT posit that self-determination is the intrapersonal (rather than interpersonal) desire to experience ownership and self-endorsement of one's behavior (Ryan \& Deci, 2000). While cultures may provide different opportunities for selfdetermination to flourish, it is nonetheless important in all cultures (Chirkov, 2009). The SDT perspective views self-determination as orthogonal, as opposed to synonymous, with individualism and independence (Vansteenkiste, Zhou, Lens, \& Soenens, 2005). These proposals have been supported by research linking self-determination with adaptive outcomes in collectivist countries, such as South Korea (Chirkov, et al., 2003), Japan (Nagasaku \& Arai, 2005); China (Vansteenkiste, et al., 2005), and Taiwan (Sheldon, et al., 2004; see also Chirkov, 2009, for a review of self-determination across cultures).

If the importance of self-determination across different cultures is to be explored within the context of $\mathrm{PE}$, then it is clear that measurement instruments must produce reliable and 
valid scores in different cultures. By establishing the validity of the PLOCQ and SIMS scores in a collectivist (HK) and individualist (UK) culture, researchers can be confident that respondents from both cultures use a similar conceptual frame of reference when completing the inventory and perceive the rating scale intervals in a similar manner (Riordan \& Vandenberg, 1994). If this is not achieved, then conclusions concerning group comparisons may be spurious (Hoyle \& Smith, 1994). To explore this issue we examined the validity of scores obtained from a sample of UK students and a sample of HK Chinese students. These two contrasting cultures provide an ideal context to examine the validity of the questionnaires across different countries. Due to immigration, colonisation, and globalisation, people in the UK and HK have undoubtedly been influenced by other cultures. However, marked differences in the cultural beliefs that these two nations hold still remain. For instance, in his seminal text on cultural dimensions, Hofstede (2001) presents compelling evidence that suggests HK and UK cultures differ in the extent of interpersonal authority a superior has over a subordinate (i.e., power distance) and the degree to which individuals associate with the collective (i.e., individualism/collectivism). Such differences have also been observed in research couched in Chinese values, rather than Western-based research, resulting in the proposal that Hofstede's work is a viable tool for selecting cultures to compare on an a priori basis (Chinese Culture Connection, 1987).

We hypothesised that the factor structure of the two questionnaires would be equivalent across cultures (within-network validity). We also predicted that relationships between PLOCQ factors and SIMS factors would be equivalent across cultures (between-network validity). 


\section{Method}

\section{Participants}

PE students $(n=392)$ from urban UK schools located in the second largest city in the UK volunteered to participate. Since children who are raised in homes in which the parents were born outside the country may be influenced by different cultural norms (McCrae, Yik, Trapnell, Bond, \& Paulhus, 1998), we removed data from students who were not born in the UK or had a parent born outside the UK. The final sample of 300 students ( $n=137$ females, $n=133$ males, and $n=30$ gender not reported) described themselves as white $(n=281)$, mixed race $(n=12)$ and black $(n=4)(n=3$ ethnicity not reported). The mean age was 13.71 years $(S D=.68$ years, range 13 to 15 years $)$.

The HK sample included 363 PE students, of whom we retained only those who were born in China and had parents who were also born in China ( $n=342 ; n=201$ females and $n=141$ male). The mean age was 15.34 years ( $S D=.54$ years, range 11 to 16 years). The students were sampled from mandatory Years 9 and 10 PE classes at three schools in the UK and mandatory Secondary 4 classes at eight schools in HK.

\section{Measures}

Contextual motivational regulations. The Perceived Locus of Causality (PLOCQ (Goudas et al., 1994) was employed to examine students' motivational regulations towards $\mathrm{PE}$ at a contextual level. Each motivational regulation comprised of four items which followed the heading "Why do you participate in Physical Education" and the stem "I take part in PE classes...”. Subscales in the questionnaire were intended to measure intrinsic motivation (e.g., 'because PE is fun'), identified regulation (e.g., 'because it is important for me to do well in PE'), introjected regulation (e.g., 'because I would feel bad about myself if I didn't'), external regulation (e.g., 'because I'll get into trouble if I don't'), and amotivation 
(e.g., 'but I don't see why we should have PE'). Responses were reported on a seven-point scale ranging from 1 (strongly disagree) to 7 (strongly agree).

Situational Motivational Regulations. A 16-item version of the Situational Intrinsic Motivation Scale (SIMS) was originally developed by Guay and his colleagues (2000). However, in three studies across diverse physical activity domains, Standage and his colleagues (2003) showed that two items produced scores that did not conform to the hypothesised factor structure. Following these authors' suggestions, a 14-item version of questionnaire was used. The item stem was "Why are you currently engaged in this activity?" and the measure included subscales designed to measure intrinsic motivation (e.g., 'Because I think that this activity is interesting'), identified regulations (e.g., 'Because I believe this activity is important for me'), external regulation (e.g., 'Because I am supposed to do it'), and amotivation (e.g., 'I do this activity but I am not sure if it is worth it'). Students responded using a seven-point scale, ranging from 1 "Not true at all" to 7 "Very true." The original version of the SIMS employed different labels (“corresponds not at all” and "corresponds exactly”); however, pilot testing in the HK Chinese sample indicated that some students found these labels confusing. As a result, we decided to employ the labels used in the PLOCQ, which the pilot sample indicated were comprehensible.

\section{Procedures}

English to Chinese translation and back translation of the questionnaires was performed using procedures advocated by van Widenfelt, Treffers, de Beurs, Siebelink, and Koudijs (2005). In particular, a translator team reached consensus on all items prior to backtranslation by an independent team. The two teams then met to resolve any discrepancies between the original and back-translated versions of the questionnaires. Following approval from a university research ethics board, informed consent was obtained from the participating schools with the teachers and/or principals acting in loco parentis, according to British 
Psychological Society ethical guidelines. The study was introduced and explained to the students at the beginning of a timetabled PE lesson. No tangible incentives (e.g., monetary reward) were offered to students and all students were given the opportunity to decline participation. Those who declined were asked to remain silent throughout the questionnaire administration. No data were collected regarding the response rate of invited students.

\section{Data Analysis}

We first tested the within-network psychometric properties of the PLOCQ and SIMS by examining the fit of the data to the hypothesised factor structure (confirmatory factor analysis [CFA] with LISREL software; Jöreskog \& Sorbom, 2004). In each CFA model, items were allowed to load on only the hypothesised factor and error terms were not allowed to correlate. $\mathrm{Hu}$ and Bentler's (1999) criteria (TLI and CFI $\geq .95, \mathrm{RMSEA} \leq .06$; $\mathrm{SRMR} \leq .08$ ) were considered to indicate good fit. We used Raykov's (1997) formula (i.e., $\left(\sum \lambda\right)^{2} /\left[\left(\sum \lambda\right)^{2}+\right.$ $\left.\left(\sum \theta\right)\right]$ ), where $\lambda=$ item-factor correlation and $\theta=$ error, to assess the internal consistency of the subscale scores. We also assessed the discriminant validity of the scales by examining the 95\% confidence intervals of the factor correlations ( $\Phi$ matrix). We followed Markland and Tobin's (2004) suggestion and investigated the presence of a simplex-like structure by examining inter-factor correlations.

We tested the cross-cultural invariance of the factor structures of scores from both the PLOCQ and SIMS using the multi-group CFA procedures outlined by Byrne (1998) and employed by Standage et al. (2003). These procedures involved first testing a baseline model with no parameter constraints (configural invariance), followed by models in which factor loadings (metric invariance), variances, covariances, and uniqueness terms were sequentially constrained to be equal across the UK and HK samples. A decrease in CFI larger than .01 
from one model to the next was taken to indicate that the more constrained model was not invariant across the samples (Cheung \& Rensvold, 2002).

Finally, we investigated 'between-network' validity by examining the covariances between contextual (PLOCQ) and situational (SIMS) motivational regulation latent factors. To accomplish this objective, we tested a CFA model that included the five PLOCQ factors and the four SIMS factors, allowing all factors to freely be estimated. This model was first tested separately in the UK and HK samples, followed by invariance testing across the samples. Examining the invariance of the covariance parameters tested the statistical significance of the differences in convergent and divergent validity in the UK and HK samples (see Introduction).

\section{Results}

\section{Reliability and Factor Structure of the PLOCQ Scores}

Descriptive statistics for individual PLOCQ items can be seen in Table 1. Preliminary analyses indicated that the data distribution for the PLOCQ scores was multivariately nonnormal (normalised multivariate skewness $=33.19$ and 24.42; normalised multivariate kurtosis $=20.88$ and 15.34 in the $\mathrm{HK}$ and UK samples, respectively). As a result, we employed robust Maximum Likelihood (ML) estimation with a Satorra-Bentler (1994) correction to the $\chi^{2}$ statistic and estimated parameter standard errors for all CFAs.

Descriptive statistics and reliability estimates for the PLOCQ scores can be seen in Table 2. The results provided support for the internal consistency of the scores derived from the amotivation, identified regulation, and intrinsic motivation subscales in both cultures (all $\rho \geq .75$ ). In the HK sample, $\rho$ of .64 and .69 emerged for the external and introjected regulation subscales, respectively. In the UK sample, the internal consistency of the scores derived from the external and introjected regulation subscales was supported with $\rho$ of .83 and .82, respectively. Despite the reliability for these two subscales being somewhat lower in 
the HK sample than in the UK sample, we decided to continue to analyse the data from the original subscales for two reasons. First, there is no firm criterion for determining the acceptability of $\rho$ (i.e., reliability is not a dichotomy of reliable vs. unreliable). Second, our purpose was to examine the psychometric properties of the scale, not to modify and improve the questionnaire.

Single group analyses indicated that, despite significant $\chi^{2}$ statistic values, the model fit the data well in the HK and UK samples (see Table 3). The interfactor correlation matrices from the single group CFA's associated with the PLOCQ scores can be seen in Table 4. We examined these matrices to investigate the discriminant validity of the factors (Anderson \& Gerbing, 1988). In both the HK and UK samples, the 95\% CI of the interfactor correlations (i.e., $\pm 1.96 \times$ standard error) associated with the identified regulation and intrinsic motivation scores encompassed 1.0. These results indicated that the identified regulation and intrinsic motivation scores were not empirically distinguishable. None of the other interfactor correlation 95\% CIs encompassed unity, thus supporting the discriminant validity of the amotivation, external regulation, and introjected regulation scores. ${ }^{1}$

\section{Simplex-like Structure of the PLOCQ Scores}

The correlation matrices in the HK and UK samples conformed to the hypothesized simplex-like structure (see Table 4). Correlations between scores intended to represent factors closer together on the self-determination continuum were stronger than the correlations between factors which were expected to be further apart on the continuum. For example, the correlation between amotivation scores and external regulation scores was stronger than the correlation between amotivation and introjected regulation scores. 


\section{Cross-Cultural Invariance of the PLOCQ Factor Structure}

The baseline multi-group model in which no constraints were placed on any of the parameters fit the data well according to the approximate fit indices (see Table 3), Testing progressively more constrained models did not result in a $\Delta \mathrm{CFI}>.01$, suggesting that the factor structure of the PLOCQ scores was invariant across the two samples.

\section{Reliability and Factor Structure of the SIMS scores}

Descriptive statistics associated with individual SIMS items can be viewed in Table 5. Preliminary analyses indicated that the data distribution for the SIMS scores was multivariately non-normal in the HK and UK samples, respectively (normalized multivariate skewness $=26.67$ and 18.95; normalized multivariate kurtosis $=22.07$ and 16.39). As a result, we employed again the robust Maximum Likelihood (ML) estimation method.

Reliability of the SIMS scores was supported in both samples ( $\rho \geq .78)$ (See Table 2). Single group analyses indicated that, despite significant $\chi^{2}$ statistic values, the SIMS model fit the data well in the HK and UK samples (see Table 6). The interfactor correlation matrices from the single group CFAs associated with the SIMS scores can be seen in Table 7. As with the PLOCQ data, the 95\% CI of the interfactor correlations associated with the SIMS identified regulation and intrinsic motivation scores encompassed 1.0 in both the UK and HK samples, suggesting that these scores were not empirically distinguishable. None of the other interfactor correlation 95\% CIs encompassed unity, thus supporting the discriminant validity of the amotivation and external regulation scores. ${ }^{2}$

\section{Simplex-like Structure of the SIMS Scores}

The correlation matrices in the HK and UK samples conformed to the hypothesized simplex-like structure. For details, see Table 7. 


\section{Cross-Cultural Invariance of the SIMS Factor Structure}

The results of the multi-group CFA's can be seen in Table 6. The baseline model, with no constrained parameters, fit the data well according to the approximate fit indices. Constraining the item-factor loadings (Model B) and variances (Model C) did not result in $\Delta \mathrm{CFI}>.01$. However, more substantial changes in CFI were seen when the covariance (Model D) estimates were constrained. These results indicated that partial measurement invariance was demonstrated (Byrne, Shavelson, \& Muthen, 1989). We conducted follow-up analyses to determine the source of the inter-sample difference. When covariances associated with the external regulation scores were freely estimated in both samples, but all other covariance terms were constrained, the model did not show a large decrease in CFI, relative to Model C. This result indicated that the external regulation scores had relationships with other factors that were not invariant across the samples (for details, see Table 6).

\section{Between-network Analyses}

The majority $(7 / 8=87.50 \%)$ of the latent factors representing identical constructs across the situational and contextual levels demonstrated the highest correlations (see Table 8). These results supported the convergent validity of the PLOCQ and SIMS latent factors. In support of divergent validity, latent factors representing motives that are theoretically more distal on the self-determination continuum were more weakly correlated. There was one notable exception to this pattern of results. In the HK sample, the correlation between SIMS external regulation and PLOCQ external regulation factors was weak and negative. Correlations between the SIMS external regulation latent factor and the other PLOCQ latent factors were higher, with the strongest positive correlations observed between the identified regulation and intrinsic motivation latent factors. ${ }^{3}$

\section{Discussion}


In this study we aimed to examine multiple facets of reliability and validity associated with scores derived from two SDT-based measures of motivation for PE. We also sought to explore cross-cultural validity. As hypothesized, and consistent with existing literature (Chatzisarantis, et al., 2003; Hagger, et al., 2005; Standage, Treasure, et al., 2003), we generally found supportive evidence of reliability as well as within- and between-network validity of scores from a contextual measure of motivation (PLOCQ) and scores from a situational measure of motivation (SIMS) specific to PE. Our findings also generally supported cross-cultural validity for the scores of the two instruments. Nonetheless, the findings illustrated some areas of concern regarding the internal consistency and factorial validity of some PLOCQ scores and the validity of SIMS scores in the HK sample.

The reliability coefficient for the introjected and external regulation scores indicated a lack of internal consistency in the items assessing contextual motivation in our HK Chinese sample. Previous findings also provide evidence for less than optimal reliability in the PLOCQ items for introjected regulation in various samples across a number of datasets gathered in both individualistic and collectivist cultures (Hagger, et al., 2005; Ntoumanis, 2005). Thus, based on our results and previous research, it appears that the items from the PLOCQ introjected regulation subscale may need to be reconsidered, with a view towards improving internal consistency of scores derived from this instrument. For example, it is possible that the current items may not be suitably consistent because they measure different aspects of introjected regulation, such as the motive to enhance contingent self-worth (e.g., "I want the teacher to think I am a good student”) and the motive to avoid low contingent selfworth (e.g., "because it bothers me when I don’t participate in PE”; Assor, Vansteenkiste, \& Kaplan, 2009). Researchers developing new items may wish to consider different aspects of introjected regulation. External regulation items from the PLOC have previously produced scores with higher internal consistency (Ntoumanis, 2001, 2005; Taylor \& Ntoumanis, 2007) 
and thus we are hesitant to recommend revision of these items. Nevertheless, further research may be needed on this issue.

Factorial validity was generally supported. Nonetheless, the self-determined motives (i.e., intrinsic motivation and identified regulation) were not distinguishable by youth in either culture. Strong relationships between identified and intrinsic motivation have been reported quite frequently in physical activity contexts. For example, studies on youth motivation using the SIMS and PLOCQ have reported observed and latent factor correlation coefficients of .73 to .99 between identified and intrinsic motivation subscale scores (Chatzisarantis, et al., 2003; Standage, Duda, \& Ntoumanis, 2005; Standage, Treasure, et al., 2003). These strong correlations suggest that youth may not differentiate between the items intended to tap identified and intrinsic regulations in PE. The strong correlations might also be due to the possibility that adolescents value behaviors that they find enjoyable and feel competent performing (Eccles \& Harold, 1991; Sabiston \& Crocker, 2008). The future development of items that enable youth to distinguish these motives within the motivational continuum may prove beneficial both for statistical and theoretical reasons. For instance, items that measure the extent to which students value the aspects of PE that are not inherently enjoyable or interesting may lead to a measure of identified regulation that is suitably discrepant from measures of intrinsic motivation.

Convergent validity was also generally supported by the findings. However, the relationships between external regulation and the other forms of motivation on the SIMS were different for students in HK and the UK, as indicated by the tests of invariance. In the UK, external regulation scores were negatively related to intrinsic motivation and identified regulation scores. These results were consistent with our hypothesis and previous research (Chatzisarantis, et al., 2003; Standage, Treasure, et al., 2003). Contrarily, in HK students, SIMS external regulation scores were positively related to SIMS intrinsic motivation and 
identified regulation scores. These results suggested that, compared to the UK, students in HK interpreted these external regulation items to be more self-determined. Importantly, this pattern of results was not observed in the PLOCQ scores. Indeed, the relationships among scores on the PLOCQ were in line with hypotheses both in the UK and HK samples. Further research is therefore needed to better understand how students from different cultures respond to SIMS items assessing external regulation. One possibility is that the emphasis placed on filial piety (i.e., showing respect for elders) in collectivist cultures may lead HK students to interpret the items measuring situational external regulations as moral obligations, rather than pure external motives. Hence, it is possible that HK students partially internalize the externally imposed motives in PE classes. It may be necessary to develop culturally appropriate items to assess this form of situation-specific motivation. These items may need to explicitly emphasize external motives, such as coercion and rewards, rather than the ambiguous terminology used in the current items.

Although this study provides general support for the validity of the two questionnaires, several future research directions can be proposed. First, within the present study we utilized procedures, such as confirmatory factor analysis, which are underpinned by classical test theory. These analyses are important, however, other approaches exist, such as procedures embedded in item-response theory (e.g., differential item functioning; Ellis \& Kimmel, 1992), that can overcome some of the problems associated with cross-cultural psychological measurement. For example, based on item-response theory's assumption of item unidimensionality, differential item functioning allows researchers to explore whether individual items are underpinned by extraneous dimensions that may create bias in a group’s responses (Hambleton, Swaminathan, \& Rogers, 1991). This type of analysis may help to explain why HK students interpreted SIMS external regulation items as more selfdetermined, compared to UK students. 
Second, researchers may wish to explore the responses of other groups of participants, such as different ethnic groups or cultural affiliations located within the same cultural context. This is particularly relevant to the UK given the ethnic diversity found in this country.

Third, the nomological validity of the questionnaires may be further examined. For example, it is of interest to explore if the different motivational regulations at the contextual and situational level predict similar behaviors (e.g., attendance in PE classes) across the two cultures. These potential future studies that may compliment the present work are important given that a second, confirmatory sample was not used within this study.

In terms of limitations, it is important to note that the specific PE-related activity in which students were engaged following the completion of the SIMS was not matched across cultures. In the Hong Kong sample students were sampled before a basketball class. In the UK sample activities varied from class to class, including trampolining, basketball, and dance. As a result, it is possible that some of the cross-cultural differences we found in the scores derived from this measure may have been due to differences in the activities that were included in the specific PE lessons. This limitation aside, our study demonstrated general measurement support in the area of motivation for physical education with the evidence of within- and between-network validity for contextual and situational measures in the UK. In other words, while some minor amendments might be needed to PLOCQ introjected regulation items, researchers interested in studying contextual and situational motivation in UK PE classes should, in general, feel confident in using the PLOCQ and the SIMS, respectively. However, our results highlight some important difficulties in the measurement of contextual and situational motivation in HK Chinese students. 


\section{Notes}

1. At the suggestion of an anonymous reviewer, we also assessed the gender invariance of the PLOCQ factor structure. The baseline model fit the data well $(\mathrm{CFI}=.98)$ and displayed no decrease in fit when all paths were constrained across genders. Indeed, the most constrained model also fit the data well (CFI $=.98)$. Contact the first author for complete details.

2. The SIMS factor structure was also invariant across genders (CFI $=.98$ in baseline and fully constrained models). Contact the first author for complete details.

3. At the suggestion of the editor, we examined the correlations between age and each of the factor scores for the PLOCQ and the SIMS. In general, these correlations were small (range $=-.16$ to .11 ), suggesting that age did not substantially influence scores. Contact the first author for complete details. 


\section{References}

Anderson, J. C., \& Gerbing, D. W. (1988). Structural equation modelling in practice: A review and recommended two-step approach. Psychological Bulletin, 102, 411-423.

Assor, A., Vansteenkiste, M., \& Kaplan, A. (2009). Identified versus introjected approach and introjected avoidance motivations in school and in sports: The limited benefits of self-worth strivings. Journal of Educational Psychology, 101, 482-497.

Byrne, B. M. (1998). Structural equation modeling with LISREL, PRELIS, and SIMPLIS. Mahwah, NJ: Erlbaum Associates.

Byrne, B. M., Shavelson, R. J., \& Muthen, B. (1989). Testing for the equivalence of factor covariance and mean structures: The issue of partial measurement invariance. Psychological Bulletin, 105, 456-466.

Chatzisarantis, N. L. D., Hagger, M. S., Biddle, S. J. H., Smith, B., \& Wang, J. C. K. (2003). A meta-analysis of perceived locus of causality in exercise, sport, and physical education contexts. Journal of Sport and Exercise Psychology, 25, 284-306.

Cheung, G. W., \& Rensvold, R. B. (2002). Evaluating goodness-of-fit indexes for testing measurement invariance. Structural Equation Modeling, 9, 233-255.

Chinese Culture Connection. (1987). Chinese values and the search for culture-free dimensions of culture. . Journal of Cross Cultural Psychology, 18, 143-164.

Chirkov, V. I. (2009). A cross-cultural analysis of autonomy in education: A selfdetermination theory perspective. Theory and Research in Education, 7, 253-262.

Chirkov, V. I., Ryan, R. M., Kim, Y., \& Kaplan, U. (2003). Differentiating autonomy from individualism and independence: A self-determination theory perspective on internalization of cultural orientations and well-being. Journal of Personality and Social Psychology, 84, 97-110. 
Deci, E. L., \& Ryan, R. M. (1985). Intrinsic motivation and self-determination in human behavior. New York: Plenum Press.

Deci, E. L., Ryan, R. M., Gagné, M., Leone, D. R., Usunov, J., \& Kornazheva, B. P. (2001). Need satisfaction, motivation, and well-being in the work organizations of a former eastern bloc country: A cross-cultural study of self-determination. Personality and Social Psychology Bulletin, 27, 930-942.

Eccles, J. S., \& Harold, R. D. (1991). Gender differences in sport involvement: Applying the Eccles' expectancy-value model. . Journal of Applied Sport Psychology, 3, 7-35.

Ellis, B. B., \& Kimmel, H. D. (1992). Identification of unique cultural response patterns by means of item-response theory. Journal of Applied Psychology, 77, 177-184.

Gordon-Larsen, P., McMurray, R. G., \& Popkin, B. M. (2000). Determinants of adolescent physical activity and inactivity patterns. Pediatrics, 105, 63-70.

Goudas, M., Biddle, S., \& Fox, K. (1994). Perceived locus of causality, goal orientations, and perceived competence in school physical education classes. British Journal of Educational Psychology, 64, 453-463.

Guay, F., Vallerand, R. J., \& Blanchard, C. (2000). On the assessment of situational intrinsic and extrinsic motivation: The situational motivation scale (SIMS). Motivation and Emotion, 24(3), 175-213.

Hagger, M. S., Chatzisarantis, N., Barkoukis, V., Wang, C. K. J., \& Baranowski, J. (2005). Perceived autonomy support in physical education and leisure-time physical activity: A cross-cultural evaluation of the trans-contextual model. Journal of Educational Psychology, 97(3), 376-390.

Hagger, M. S., Chatzisarantis, N. L. D., Culverhouse, T., \& Biddle, S. J. H. (2003). The processes by which perceived autonomy support in physical education promotes 
leisure-time physical activity intentions and behavior: A trans-contextual model. Journal of Educational Psychology, 95(4), 784-795.

Hambleton, R. K., Swaminathan, H., \& Rogers, H. J. (1991). Fundamentals of item response theory. Newbury Park, CA: Sage Publications.

Hofstede, G. (2001). Cultures consequences: Comparing values, behaviors, institutions, and organizations across nations ( $2^{\text {nd }}$ Ed. ed.). Thousand Oaks, CA: Sage.

Hoyle, R. H., \& Smith, G. T. (1994). Formulating clinical research hypotheses as structural equation models: A conceptual overview. Journal of Consulting and Clinical Psychology, 62, 429-440.

Hu, L.-t., \& Bentler, P. M. (1999). Cutoff criteria for fit indexes in covariance structure analysis: Conventional criteria versus new alternatives. Structural Equation Modeling, 6(1), 1-55.

Iyengar, S. S., \& Lepper, M. R. (1999). Rethinking the value of choice: A cultural perspective on intrinsic motivation. Journal of Personality and Social Psychology, 76(3), 349-366.

Jöreskog, K. G., \& Sorbom, D. (2004). LISREL 8.71 [Computer software]. Lincolnwood, IL: Scientific Software International, Inc.

Lonsdale, C., Sabiston, C. M., Raedeke, T. D., Ha, S. C. A., \& Sum, K. W. R. (2009). Selfdetermined motivation and students' physical activity in PE classes and free-choice periods. Preventive Medicine, 48, 69-73.

Markland, D., \& Tobin, V. (2004). A modification to the Behavioural Regulation in Exercise Questionnaire to include an assessment of amotivation. Journal of Sport \& Exercise Psychology, 26, 191-196.

Markus, H. R., \& Kitayama, S. (1991). Culture and the self: Implications for cognition, emotion, and motivation. Psychological Review, 98(2), 224-253. 
Marsh, H. W. (1998). Foreword. In J. L. Duda (Ed.), Advances in sport and exercise psychology measurement (pp. xv-xix). Morgantown, WV: Fitness Information Technology.

McCrae, R. R., Yik, M. S., Trapnell, P. D., Bond, M. H., \& Paulhus, D. L. (1998). Interpreting personality profiles across cultures: Bilingual, acculturation, and peer rating studies of Chinese undergraduates. Journal of Personality and Social Psychology, 74(4), 1041-1055.

Messick, S. (1995). Validity of psychological assessment: Validation of inferences from persons' responses and performances as scientific inquiry into score meaning. American Psychologist, 50(9), 741-749.

Nagasaku, M., \& Arai, K. (2005). Autonomous motivation to enter high school and school adjustment: A short-term longitudinal study. Japanese Journal of Educational Psychology, 53(516-528).

Ntoumanis, N. (2001). A self-determination approach to the understanding of motivation in physical education. British Journal of Educational Psychology, 71(2), 225-242.

Ntoumanis, N. (2005). A prospective study of participation in optional school physical education using a self-determination theory framework. Journal of Educational Psychology, 97(3), 444-453.

Ntoumanis, N., \& Standage, M. (2009). Motivation in physical education classes: A selfdetermination theory perspective. Journal of Research and Theory in Education, 7, 194-202.

Prusak, K. A., Treasure, D. C., Darst, P. W., \& Pangrazi, R. P. (2004). The effects of choice on the motivation of adolescent girls in physical education. Journal of Teaching in Physical Education, 23, 19-29. 
Riordan, C. M., \& Vandenberg, R. J. (1994). A central question in cross-cultural research: Do employees of different cultures interpret work-related measures in an equivalent manner. Journal of Management, 20, 643-671.

Ryan, R. M., \& Connell, J. P. (1989). Perceived locus of causality and internalization: Examining reasons for acting in two domains. Journal of Personality \& Social Psychology, 57(5), 749-761.

Ryan, R. M., \& Deci, E. L. (2002). Overview of self-determination theory: An organismic dialectical perspective. In E. L. Deci \& R. M. Ryan (Eds.), Handbook of selfdetermination research (pp. 3-33). Rochester, NY: The University of Rochester Press.

Sabiston, C. M., \& Crocker, P. R. E. (2008). Exploring a model of self-perceptions and social influences in the prediction of adolescent leisure-time physical activity. . Journal of Sport and Exercise Psychology, 30, 3-22.

Satorra, A., \& Bentler, P. M. (1994). Corrections to test statistic and standard errors in covariance structure analysis. In A. v. Eye \& C. C. Clogg (Eds.), Latent variables analysis: Applications for developmental research (pp. 399-419). Thousand Oaks, CA: Sage.

Sheldon, K. M., Elliot, A. J., Ryan, R. M., Chirkov, V., Kim, Y., Wu, C., et al. (2004). Selfconcordance and subjective well-being in four cultures. Journal of Cross-cultural psychology, 35(2), 209-223.

Standage, M., Duda, J. L., \& Ntoumanis, N. (2003). A model of contextual motivation in physical education: Using constructs from self-determination and achievement goal theories to predict physical activity intentions. Journal of Educational Psychology, 95, 97-110. 
Standage, M., Duda, J. L., \& Ntoumanis, N. (2005). A test of self-determination theory in school physical education. British Journal of Educational Psychology, 75(3), 411433.

Standage, M., Treasure, D. C., Duda, J. L., \& Prusak, K. A. (2003). Validity, reliability, and invariance of the Situational Motivation Scale (SIMS) across diverse physical activity contexts. Journal of Sport \& Exercise Psychology, 25(1), 19-43.

Taylor, I. M., \& Ntoumanis, N. (2007). Teacher motivational strategies and student selfdetermination in physical education Journal of Educational Psychology, 99 747-760.

Vallerand, R. J. (2001). A heirarchical model of intrinsic and extrinsic motivation in sport and exercise. In G. C. Roberts (Ed.), Advances in motivation in sport and exercise. Champaign, IL: Human Kinetics.

Vallerand, R. J., Pelletier, L. G., Blais, M. R., Briere, N. M., Senecal, C., \& Vallieres, E. F. (1992). The Academic Motivation Scale: A measure of intrinsic, extrinsic, and amotivation in education. Educational \& Psychological Measurement, 52, 1003-1017.

van Widenfelt, B. M., Treffers, P. D. A., de Beurs, E., Siebelink, B. M., \& Koudijs, E. (2005). Translation and cross-cultural adaptation of assessment instruments used in psychological research with children and families. Clinical Child and Family Psychology Review, 8(2), 135-147.

Vansteenkiste, M., Zhou, M., Lens, W., \& Soenens, B. (2005). Experiences of autonomy and control among Chinese learners: Vitalizing or immobilizing? Journal of Educational Psychology, 97, 468-483.

Wang, C. K. J., \& Liu, W. C. (2007). Promoting enjoyment in girls' physical education: The impact of goals, beliefs, and self-determination. European Physical Education Review, 13, 145-164. 
Table 1

Univariate Normality, Item-Factor Loadings and Error Terms from Confirmatory Factor Analysis of PLOCQ Responses

\begin{tabular}{|c|c|c|c|c|c|c|c|c|}
\hline \multirow[b]{2}{*}{ Subscale } & \multicolumn{4}{|c|}{ Hong Kong } & \multicolumn{4}{|c|}{ United Kingdom } \\
\hline & Skew $(Z)$ & Kurtosis (Z) & $\begin{array}{l}\text { Item-Factor } \\
\text { Loading }\end{array}$ & Error & Skew $(Z)$ & Kurtosis (Z) & $\begin{array}{l}\text { Item- } \\
\text { Factor } \\
\text { Loading }\end{array}$ & Error \\
\hline Amotivation & & & & & & & & \\
\hline but I really don’t know why. & $.04(.31)$ & $-.21(-.81)$ & .56 & .69 & $.43(3.07)$ & $-.97(-3.46)$ & .60 & .64 \\
\hline but I don't see why we should have PE. & $.18(1.38)$ & $-.11(-.42)$ & .62 & .62 & $.63(4.50)$ & $-.96(-3.43)$ & .81 & .34 \\
\hline but I really feel I'm wasting my time in PE. & $.03(.23)$ & $-.43(-1.65)$ & .73 & .47 & $.67(4.79)$ & $-.84(-3.00)$ & .89 & .21 \\
\hline but I don't see what I get out of PE & $.34(2.62)$ & $.02(.08)$ & .73 & .47 & $.56(4.00)$ & $-.89(-3.18)$ & .86 & .27 \\
\hline External Regulation & & & & & & & & \\
\hline because I'll get into trouble if I don't. & $.34(2.62)$ & $-.80(-3.08)$ & .59 & .65 & $-.17(-1.21)$ & $-1.12(-4.00)$ & .67 & .55 \\
\hline because that's what I am supposed to do. & $.36(2.77)$ & $-.40(-1.54)$ & .10 & .99 & $-.28(-2.00)$ & $-.86(-3.07)$ & .60 & .64 \\
\hline so that the teacher won't yell at me. & $.16(1.23)$ & $-.16(-.62)$ & .70 & .51 & $.32(2.29)$ & $-1.27(-4.54)$ & .77 & .41 \\
\hline because that's the rule. & $-.02(-.15)$ & $-.26(-1.00)$ & .56 & .68 & $-.09(-.64)$ & $-1.13(-4.04)$ & .71 & .49 \\
\hline
\end{tabular}




\begin{tabular}{|c|c|c|c|c|c|c|c|c|}
\hline Introjected Regulation & & & & & & & & \\
\hline $\begin{array}{l}\text { because I want the teacher to think I'm a } \\
\text { good student. }\end{array}$ & $.18(1.38)$ & $-.32(-1.23)$ & .53 & .72 & $-.01(-.07)$ & $-.78(-2.79)$ & .53 & .72 \\
\hline because I would feel guilty if I didn’t. & $.03(.23)$ & $-.53(-2.04)$ & .52 & .74 & $.73(5.21)$ & $-.31(-1.11)$ & .71 & .50 \\
\hline $\begin{array}{l}\text { because I would feel bad about myself if I } \\
\text { didn’t. }\end{array}$ & $.31(2.38)$ & $-.53(-2.04)$ & .58 & .67 & $.41(2.93)$ & $-.86(-3.07)$ & .64 & .60 \\
\hline $\begin{array}{c}\text { because it bothers me when I don’t. } \\
\text { Identified Regulation }\end{array}$ & $-.04(-.31)$ & $-.44(-1.69)$ & .55 & .70 & $.55(3.93)$ & $-.71(-2.54)$ & .76 & .42 \\
\hline because I want to learn sport skills. & $.31(2.38)$ & $-.55(-2.12)$ & .70 & .51 & $-.52(-3.71)$ & $-.73(-2.61)$ & .87 & .24 \\
\hline $\begin{array}{l}\text { because it is important for me to do well in } \\
\text { PE. }\end{array}$ & $.01(.08)$ & $-.44(-1.69)$ & .65 & .58 & $-.30(-2.14)$ & $-.96(-3.43)$ & .81 & .35 \\
\hline because I want to improve in PE. & $-.57(-4.38)$ & $-.09(-.35)$ & .71 & .49 & $-.50(-3.57)$ & $-.90(-3.21)$ & .86 & .27 \\
\hline $\begin{array}{l}\text { because I can learn skills which I could use } \\
\text { in other areas of my life. }\end{array}$ & $-.40-3.08)$ & $.07(-.27)$ & .55 & .70 & $-.35(-2.50)$ & $-.90(-3.21)$ & .74 & .45 \\
\hline Intrinsic Motivation & & & & & & & & \\
\hline $\begin{array}{l}\text { because PE is fun. } \\
\text { because I enjoy learning new skills. }\end{array}$ & $\begin{array}{l}-.01(-.08) \\
.02(.15)\end{array}$ & $\begin{array}{l}-.47(-1.81) \\
.09(.35)\end{array}$ & $\begin{array}{l}.78 \\
.69\end{array}$ & $\begin{array}{l}.39 \\
.53\end{array}$ & $\begin{array}{l}-.38(-2.71) \\
-.41(-2.93)\end{array}$ & $\begin{array}{l}-1.07(-3.82) \\
-.84(-3.00)\end{array}$ & $\begin{array}{l}.89 \\
.85\end{array}$ & $\begin{array}{l}.22 \\
.27\end{array}$ \\
\hline because PE is exciting. & $.24(1.85)$ & $-.09(-.35)$ & .78 & .40 & $-.11(-.79)$ & $-1.17(-4.18)$ & .85 & .27. \\
\hline $\begin{array}{l}\text { because of the enjoyment I feel when } \\
\text { learning new skills. }\end{array}$ & $-.41(-3.15)$ & $-.22(-.85)$ & .69 & .52 & $-.11(-.79)$ & $-1.14(-4.07)$ & .78 & .39 \\
\hline
\end{tabular}


Table 2

Internal Consistency and Descriptive Statistics of PLOCQ and SIMS Subscale Scores

\begin{tabular}{|c|c|c|c|c|c|c|c|c|}
\hline \multirow[b]{3}{*}{ Subscale } & \multicolumn{4}{|c|}{ PLOCQ } & \multicolumn{4}{|c|}{ SIMS } \\
\hline & \multicolumn{2}{|c|}{ United Kingdom } & \multicolumn{2}{|c|}{ Hong Kong } & \multicolumn{2}{|c|}{ United Kingdom } & \multicolumn{2}{|c|}{ Hong Kong } \\
\hline & $\rho$ & $M(S D)$ & $\rho$ & $M(S D)$ & $\rho$ & $M(S D)$ & $\rho$ & $M(S D)$ \\
\hline Amotivation & .87 & $2.43(1.13)$ & .76 & $2.58(.81)$ & .85 & $3.28(1.75)$ & .78 & 3.44 (1.13) \\
\hline $\begin{array}{l}\text { External } \\
\text { Regulation }\end{array}$ & .83 & $3.02(1.02)$ & .64 & $3.03(.66)$ & .78 & $4.25(1.61)$ & .83 & 3.99 (1.15) \\
\hline $\begin{array}{l}\text { Introjected } \\
\text { Regulation }\end{array}$ & .82 & $2.56(.91)$ & .69 & $2.74(.60)$ & - & - & - & - \\
\hline $\begin{array}{l}\text { Identified } \\
\text { Regulation }\end{array}$ & .89 & $3.44(1.10)$ & .75 & $3.40(.66)$ & .82 & $4.37(1.66)$ & .79 & 3.88 (1.09) \\
\hline $\begin{array}{l}\text { Intrinsic } \\
\text { Motivation }\end{array}$ & .92 & $3.28(1.17)$ & .86 & $3.49(.77)$ & .92 & $4.31(1.75)$ & .93 & $4.00(1.30)$ \\
\hline
\end{tabular}

Note: PLOCQ scores were measured on a five-point scale. SIMS scores were measured on a seven-point scale. The SIMS does not contain an introjected regulation subscale. $\rho=(\Sigma \lambda)^{2} /\left[(\Sigma \lambda)^{2}+(\Sigma \theta)\right]$, where $\lambda=$ item-factor correlation and $\theta=$ error. 
Table 3

CFA PLOCQ Model Fit Statistics

\begin{tabular}{lcccccccc}
\hline \multicolumn{1}{c}{ Model (new constraint) } & df & SB $\chi^{2}$ & CFI & $\Delta$ CFI & TLI & RMSEA & $\begin{array}{c}\text { RMSEA } \\
\text { S0\% CI }\end{array}$ & SRMR \\
\hline UK sample & 160 & 378.38 & .98 & - & .98 & .06 & $.05-.08$ & .09 \\
HK sample & 160 & 316.07 & .97 & - & .96 & .05 & $.04-.06$ & .08 \\
MG-CFA Model A (Baseline- & & & & & & & & \\
No Constraint) & 320 & 971.83 & .96 & - & .95 & .08 & $.07-.08$ & .09 \\
Model B (Loadings) & & & & & & & .09 & .09 \\
Model C (Variances) & 335 & 1050.39 & .96 & .00 & .95 & .08 & $.07-.09$ & .09 \\
Model D (Covariances) & 340 & 1156.31 & .95 & .00 & .94 & .09 & $.08-.09$ & .10 \\
Model E (Uniqueness) & 350 & 1194.06 & .95 & .00 & .94 & .09 & $.08-.09$ & .10 \\
\hline
\end{tabular}

Note: Dashes indicate that the particular statistics were not applicable. Models A through E represent progressively more constrained multi-group (MG) CFA models. Each new constraint is listed in parentheses beside the model in which it was first added. $\triangle$ CFI values were calculated from CFI values accurate to three decimal places whereas CFI values shown in this table have been rounded to two decimal places. 
Table 4

Correlations Among PLOCQ Latent Factor Scores ( $\Phi$ matrix) in the United Kingdom (Below the Diagonal) and Hong Kong (Above the Diagonal) Samples

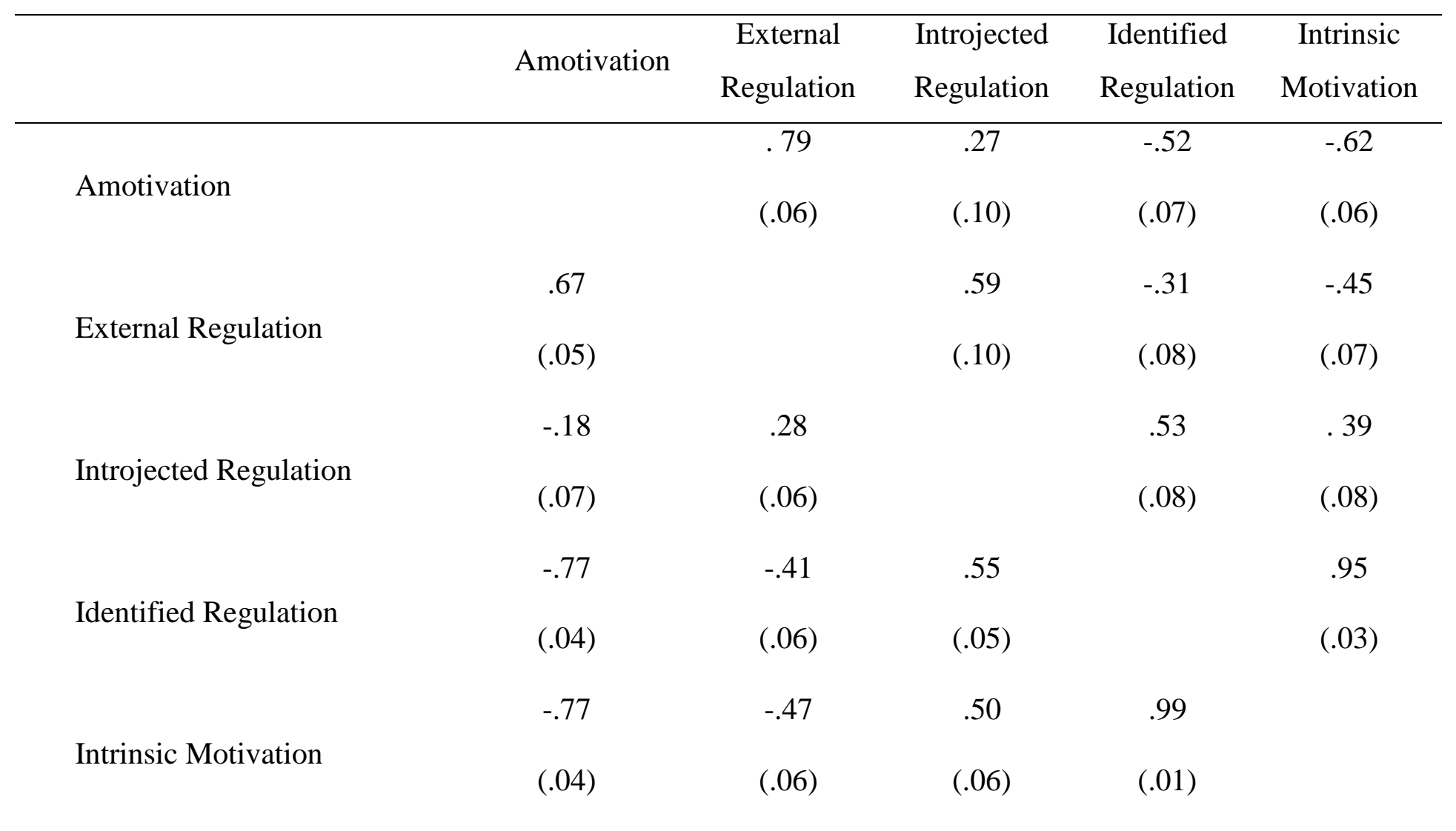

Note: All correlations are significant at $p<.05$. Standard errors are listed in parentheses. $\Phi=$

Phi (i.e., latent score correlations). 
Table 5

Univariate Normality, Item-Factor Loadings and Error Terms from Confirmatory Factor Analysis of SIMS Responses

\begin{tabular}{|c|c|c|c|c|c|c|c|c|}
\hline & \multicolumn{4}{|c|}{ Hong Kong } & \multicolumn{4}{|c|}{ United Kingdom } \\
\hline & Skew $(Z)$ & Kurtosis (Z) & $\begin{array}{l}\text { Item- } \\
\text { Factor } \\
\text { Loading }\end{array}$ & Error & Skew $(Z)$ & Kurtosis $(Z)$ & $\begin{array}{l}\text { Item- } \\
\text { Factor } \\
\text { Loading }\end{array}$ & Error \\
\hline \multicolumn{9}{|l|}{ Amotivation } \\
\hline $\begin{array}{l}\text { There may be good reasons to do this activity, but } \\
\text { personally I don't see any. }\end{array}$ & $.46(3.54)$ & $.19(.73)$ & .58 & .66 & $.46(3.29)$ & $-.92(-3.29)$ & .81 & .35 \\
\hline I do this activity but I am not sure if it is worth it. & $.33(2.54)$ & $.12(.46)$ & .76 & .43 & $.55(3.93)$ & $-.68(-2.43)$ & .75 & .44 \\
\hline $\begin{array}{l}\text { I don't know; I don't see what the activity brings } \\
\text { me. }\end{array}$ & $.46(3.54)$ & .35 (1.35) & .79 & .38 & $.63(4.50)$ & $-.61(-2.18)$ & .84 & .29 \\
\hline $\begin{array}{l}\text { I do this activity, but I am not sure it is a good } \\
\text { thing to pursue it. }\end{array}$ & $.34(2.62)$ & $.65(2.50)$ & .61 & .63 & $.45(3.21)$ & $-.88(-3.14)$ & .65 & .57 \\
\hline \multicolumn{9}{|l|}{ External Regulation } \\
\hline Because I am supposed to do it. & $-.04(-.31)$ & $.31(1.19)$ & .79 & .38 & $-.14(-1.00)$ & $-1.13(-4.04)$ & .77 & .41 \\
\hline Because it is something that I have to do. & $-.02(-.15)$ & $.18(.69)$ & .78 & .39 & $-.11(-.79)$ & $-1.05(-3.75)$ & .73 & .46 \\
\hline Because I feel that I have to do it. & $-.001(.01)$ & $.35(1.35)$ & .78 & .39 & $.12(.86)$ & $-1.08(-3.86)$ & .71 & .50 \\
\hline \multicolumn{9}{|l|}{ Identified Regulation } \\
\hline Because I am doing it for my own good. & $.18(1.38)$ & $-.18(-.69)$ & .59 & .65 & $-.15(-1.07)$ & $-1.06(-3.79)$ & .73 & .47 \\
\hline Because I think this activity is good for me. & $.12(.92)$ & $.17(.65)$ & .83 & .32 & $-.41(-2.93)$ & $-.87(-3.11)$ & .86 & .26 \\
\hline Because I feel that I have to do it. & $.18(1.38)$ & $.19(.73)$ & .81 & .34 & $-.17(-1.21)$ & $-1.08(-3.86)$ & .74 & .45 \\
\hline \multicolumn{9}{|l|}{ Intrinsic Motivation } \\
\hline Because I think that this activity is interesting. & $.24(1.85)$ & $-.26(-1.00)$ & .87 & .24 & $-.06(-.43)$ & $-1.23(-4.39)$ & .87 & .24 \\
\hline Because I think that this activity is pleasant. & $.16(1.23)$ & $.01(.04)$ & .89 & .21 & $-.09(-.64)$ & $-1.05(-3.75)$ & .88 & .22 \\
\hline Because this activity is fun. & $.13(1.00)$ & $-.35(-1.35)$ & .90 & .20 & $-.28(-2.00)$ & $-1.23(-4.39)$ & .88 & .22 \\
\hline Because I feel good when doing this activity. & $.06(.46)$ & $.31(1.19)$ & .86 & .27 & $-.20(-1.43)$ & $-1.00(-3.57)$ & .82 & .32 \\
\hline
\end{tabular}


Table 6

CFA SIMS Model Fit Statistics

\begin{tabular}{lcccccccc}
\hline \multicolumn{1}{c}{\begin{tabular}{c} 
Model \\
\multicolumn{1}{c}{ (new constraint) }
\end{tabular}} & $\mathrm{df}$ & $\mathrm{SB} \chi^{2}$ & $\mathrm{CFI}$ & $\Delta$ CFI & TLI & RMSEA & RMSEA 90\% CI & SRMR \\
\hline UK Sample & 71 & 156.53 & .99 & - & .99 & .05 & $.04-.06$ & .05 \\
HK Sample & 71 & 155.08 & .99 & - & .99 & .05 & $.04-.06$ & .06 \\
MG-CFA Model A( Baseline- & 142 & 467.39 & .97 & - & .96 & .08 & $.08-.09$ & .05 \\
No Constraint) & & & & & & & .05 \\
Model B (Loadings) & 152 & 482.46 & .97 & -.01 & .96 & .08 & $.07-.09$ & .05 \\
Model C (Variances) & 156 & 541.15 & .96 & -.01 & .96 & .09 & $.08-.09$ & .09 \\
Model D (Covariances) & 162 & 708.75 & .95 &.-02 & .94 & .10 & $.09-.11$ & .21 \\
Model E Uniqueness & 176 & 1336.03 & .89 & -.05 & .89 & .14 & $.13-.15$ & .25
\end{tabular}

Note: Dashes indicate that the particular statistics were not applicable. Models A through E

represent progressively more constrained multi-group (MG) CFA models. Each new constraint

is listed in parentheses beside the model in which it was first added. $\Delta$ CFI values were

calculated from CFI values accurate to three decimal places whereas CFI values shown in this

table have been rounded to two decimal places. 
Table 7

Correlations Between SIMS Latent Factor Scores ( $\Phi$ matrix) in United Kingdom (Below the Diagonal) and Hong Kong (Above the Diagonal) Samples

\begin{tabular}{lcccc}
\hline & Amotivation & External & External & Intrinsic \\
& & Regulation & Regulation & Motivation \\
\hline Amotivation & & -.19 & -.51 & -.48 \\
External Regulation & .59 & $(.07)$ & $(.07)$ & $(.09)$ \\
& $(.18)$ & & .73 & .58 \\
Identified Regulation & -.51 & -.08 & $(.10)$ & $(.11)$ \\
& $(.16)$ & $(.14)$ & & .96 \\
Intrinsic Motivation & -.49 & -.21 & .95 & $(.12)$ \\
& $(.17)$ & $(.14)$ & $(.17)$ &
\end{tabular}

Note: The underlined correlation was not statistically significant. All other correlations are significant at $p<.05$.

Standard errors are listed in parentheses. $\Phi=$ Phi (i.e., latent score correlations). 
Table 8

Correlations Between PLOCQ and SIMS Latent Factor Scores ( $\Phi$ matrix)

\begin{tabular}{|c|c|c|c|c|c|c|c|c|c|c|}
\hline & \multicolumn{5}{|c|}{ United Kingdom } & \multicolumn{5}{|c|}{ Hong Kong } \\
\hline & \multicolumn{10}{|c|}{ PLOC Factors } \\
\hline $\begin{array}{l}\text { SIMS } \\
\text { factors }\end{array}$ & $\begin{array}{c}\text { Intrinsic } \\
\text { Motv. }\end{array}$ & $\begin{array}{c}\text { Identified } \\
\text { Reg. }\end{array}$ & $\begin{array}{c}\text { Introjected } \\
\text { Reg. }\end{array}$ & $\begin{array}{c}\text { External } \\
\text { Reg. }\end{array}$ & $\begin{array}{l}\text { Amot- } \\
\text { ivation }\end{array}$ & $\begin{array}{c}\text { Intrinsic } \\
\text { Motv. }\end{array}$ & $\begin{array}{c}\text { Identified } \\
\text { Reg. }\end{array}$ & $\begin{array}{c}\text { Introjected } \\
\text { Reg. }\end{array}$ & $\begin{array}{c}\text { External } \\
\text { Reg. }\end{array}$ & $\begin{array}{l}\text { Amot- } \\
\text { ivation }\end{array}$ \\
\hline Intrinsic & .76 & .68 & .36 & -.28 & -.55 & .54 & .46 & .11 & -.27 & -.33 \\
\hline Motivation & $(.04)$ & $(.04)$ & $(.06)$ & $(.06)$ & $(.05)$ & $(.05)$ & $(.06)$ & $(.09)$ & $(.07)$ & $(.07)$ \\
\hline Identified & .76 & .76 & .47 & -.19 & -.61 & .55 & .54 & .15 & -.17 & -.30 \\
\hline Regulation & (.04) & (.03) & $(.06)$ & $(.06)$ & $(.05)$ & $(.06)$ & $(.06)$ & (.09) & $(.08)$ & $(.07)$ \\
\hline External & -.14 & $\underline{-.09}$ & .22 & .65 & .30 & .48 & .51 & .24 & -.18 & -.24 \\
\hline Regulation & $(.07)$ & $(.07)$ & $(.07)$ & $(.05)$ & $(.07)$ & $(.07)$ & $(.07)$ & $(.10)$ & $(.08)$ & $(.08)$ \\
\hline & -.54 & -.52 & -.10 & .46 & .76 & -.40 & -.28 & .18 & .41 & .58 \\
\hline & $(.05)$ & $(.05)$ & $(.06)$ & $(.05)$ & $(.04)$ & $(.07)$ & $(.08)$ & (.10) & $(.08)$ & $(.07)$ \\
\hline
\end{tabular}

Note. Reg. = Regulation. Motv = Motivation. Boldface coefficients represent relationships that were hypothesized to be stronger than other relationships between factor scores. The SIMS does not contain a scale to measure introjected regulation. Standard errors are listed in parentheses. Underlined correlations were not significant at $p<.05$. All other correlations were significant at $p<.05$. Tests of invariance revealed no significant difference in the models comparing the covariances between the UK and HK samples. $\Phi=$ Phi (i.e., latent score correlations). 